
a) Freie Mitteilungen
Gynaecologia 1962;153:81

\title{
ACTH-Behandlung bei schwerem Schwangerschafts- erbrechen

\begin{tabular}{|l|l|l|}
\hline H. & & Erb \\
\hline M. & Keller \\
\hline
\end{tabular}

Basel

Erschienen in Gynaecologia 152: 258-264 (1961).

Diskussion

F. Roth (Bern): Es ware interessant zu wissen, ob sich bei Ihren schweren Hyperemesisfällen auch Frauen befanden, die schon Leuzin- und Tyrosinkristalle im Urinsediment aufwiesen. Das Vorhandensein dieser Kristalle deutet auf eine so schwere Stoffwechselstörung hin, daß die Unterbrechung der Schwangerschaft jeweils gerechtfertigt ist. Sollte nun die ACTH-Therapie auch in solchen Fallen wirksam sein, ware das als großer Fortschritt zu werten.

H. Erb und M. Keller (Basel): Praktisch bei alien unseren Hyperemesisfällen wurde die Aminosäure-Ausscheidung papierchromatographisch kontroUiert. Wir fanden nie Anhaltspunkte für eine vermehrte Leuzin- oder Tyrosinausscheidung.

6 Gynaecologia, Vol. 153, No. 2 (1962)

Schweiz. Ges. Geb. Gynäk., Jahresvers. 1961, Interlaken. Gynaecologia 153: 82-84 (1962)

Aus der Universitäts-Frauenklinik Zurich (Direktor: Prof. Dr. E. Held)

Mortalîtät von Mutter und Kind bei unsern Spätgestosen

Von K. FRIES

Zur Berechnung der kindlichen und mütterlichen Mortalîtät unse-rer Spätgestosen werteten wir die Fälle der Jahre 1952-60 nach dem Lochkartenverfahren aus. Dabei wurden die

Krankengeschichten schon während der Hospitalisation der Mutter hinsichtlich der Ana-mnese durch Rückfragen bei Arzten und Kliniken, bei denen die Patientin früher in Behandlung stand, sorgfältig komplettiert; die Untersuchungen, die Therapie und die Nachkontrollen nach festgelegten Richtlinien durchgeführt.

Die Einteilung erfolgte entsprechend der Schwere der Symptoma-tik in Anlehnung an das bekannte Schema von Díeckmann, wobei wir aber die Prae-Eklampsien als besondere Gruppe innerhalb der genui-nen Spätgestosen herausgeschält haben. Dies erscheint uns wegen der Dramatik des klinischen Bildes und wegen der schlechten Prognose für das Kind gerechtfertigt. Von insgesamt 1706 Spätgestosen (5,48\% des Gesamtgeburten-materials ausmachend) kamen 216 Kinder von mehr als 30 cm Kör-perlänge ad exitum. Dies macht eine Gesamtmortalität von 12,66 \% aus und ist rund 3mal so hoch wie die jährliche kindliche Mortalîtät an unserer Klinik. Die gereinigte Mortalîtät (d. h. nach Abzug der unreifen Kinder und lebensunfähigen Mißbildungen) beträgt noch 9,2 \%. Die prozentuale Verteilung auf die einzelnen Grade der Spätgestosen ist die folgende: leichte Spätgestosen 7,78\%, mittel-schwere 11,04\%, schwere 10,15\%; Prae-Eklampsien 28,00\%, Eklampsien 35,59\% und Pfropfgestosen 21,78\%. 
35,2 \% unserer kindlichen Todesfälle waren schon vor Klinikein-tritt intrauterin abgestorben und damit einer klinischen Therapie entzogen. Eine Spitalbehandlung von mehr als 2 Tagen bis zum Ein-tritt des Todes oder der Geburt konnte nur bei 12,4 \% aller 216 Fälle mit totem Kind durchgeführt werden.

Bei der Aufschlüsselung unserer Todesfälle konnten wir

kurz zu-sammengefaßt

folgende typischen Daten ermitteln: Die Tragze.it ist 